\title{
Development of Resident-Sensitive Quality Measures for Inpatient General Internal Medicine
}

\author{
Benjamin Kinnear, MD, MEd ${ }^{1,2}{ }^{0}$, Matthew Kelleher, MD, MEd ${ }^{1,2}$, Dana Sall, MD, MEd?, \\ Daniel P. Schaver, MD, MSc'², Eric J. Warm, MD², Andrea Kachelmeyer, $B S^{7}$, \\ Abigail Martini, $B S^{7}$, and Daniel J. Schumacher, $M D, P h D^{7}$
}

'Department of Pediatrics, University of Cincinnati College of Medicine, Cincinnati, OH, USA; ${ }^{2}$ Department of Internal Medicine, University of Cincinnati College of Medicine, Cincinnati, OH, USA.

\begin{abstract}
BACKGROUND: Graduate medical education (GME) training has long-lasting effects on patient care quality. Despite this, few GME programs use clinical care measures as part of resident assessment. Furthermore, there is no gold standard to identify clinical care measures that are reflective of resident care. Resident-sensitive quality measures (RSQMs), defined as "measures that are meaningful in patient care and are most likely attributable to resident care," have been developed using consensus methodology and piloted in pediatric emergency medicine. However, this approach has not been tested in internal medicine (IM).
\end{abstract}

OBJECTIVE: To develop RSQMs for a general internal medicine (GIM) inpatient residency rotation using previously described consensus methods.

DESIGN: The authors used two consensus methods, nominal group technique (NGT) and a subsequent Delphi method, to generate RSQMs for a GIM inpatient rotation. RSQMs were generated for specific clinical conditions found on a GIM inpatient rotation, as well as for general care on a GIM ward.

PARTICIPANTS: NGT participants included nine IM and medicine-pediatrics (MP) residents and six IM and MP faculty members. The Delphi group included seven IM and MP residents and seven IM and MP faculty members. MAIN MEASURES: The number and description of RSQMs generated during this process.

KEY RESULTS: Consensus methods resulted in 89 RSQMs with the following breakdown by condition: GIM general care-21, diabetes mellitus-16, hyperkalemia-14, COPD-13, hypertension-11, pneumonia-10, and hypokalemia-4. All RSQMs were process measures, with $48 \%$ relating to documentation and $51 \%$ relating to orders. Fifty-eight percent of RSQMs were related to the primary admitting diagnosis, while $42 \%$ could also be related to chronic comorbidities that require management during an admission.

CONCLUSIONS: Consensus methods resulted in 89 RSQMs for a GIM inpatient service. While all RSQMs were process measures, they may still hold value in learner assessment, formative feedback, and program evaluation.

KEY WORDS: assessment; graduate medical education; competencybased medical education; quality.

Received May 18, 2020

Revised July 20, 2020

Accepted October 14, 2020

Published online October 26, 2020
J Gen Intern Med 36(5):1271-8

DOI: $10.1007 / \mathrm{s} 11606-020-06320-0$

(C) Society of General Internal Medicine 2020

\section{INTRODUCTION}

Graduate medical education (GME) training has long-lasting effects on the quality of care that graduates deliver. ${ }^{1-5}$ However, these "imprinting" studies focus mainly on clinical performance measures (CPMs) of former residents ${ }^{6}$ and not on measures during residency. This gap has led some to advocate for CPMs in trainee assessment that could determine readiness for graduation or predict performance after training. ${ }^{7}$ Without this, program directors do not truly know if their educational outcomes connect with quality care at the heart of GME's mission.

Ideally, GME programs could easily measure CPMs that are attributable to resident care. This could enhance feedback to learners, provide accountability to the public, improve program evaluation, and justify funding of education research. ${ }^{8}$ While some programs have started bridging the gap between CPMs and resident assessment, ${ }^{9-13}$ most face barriers, including lack of defined and agreed upon CPMs, difficulty with attribution of care to individuals, variable case-mix indices, small sample sizes, and data transparency and confidentiality issues. ${ }^{8,14,15}$

The growing calls for connecting education to $\mathrm{CPMs}^{16-22}$ have led to promising ideas. Haan et al. used a tiered framework to identify care measures that could be used to measure education quality. ${ }^{11}$ Kalet et al. and Yin et al. used theoretical models to identify "educationally sensitive patient outcomes," defined as meaningful patient outcomes that are sensitive to provider education. ${ }^{22,23}$ Building on these studies, Schumacher et al. used consensus-building methods to identify "resident-sensitive quality measures" (RSQMs), defined as measures that are meaningful in patient care and mostly attributable to resident care. ${ }^{24,25}$ RSQMs were initially developed and implemented in pediatric emergency medicine (PEM), showing discrimination between learners across patient encounters. ${ }^{26-28}$ While the initial study used National Quality Measures Clearinghouse measures as a starting point 
for measure development, the authors found that relatively few measures met criteria to be RSQMs. ${ }^{24}$

Most workplace-based assessment (WBA) approaches suffer from unwanted rater effects such as variable frames of reference, high levels of inference, differing methods for synthesizing judgments into numerical ratings, and constructirrelevant variance. ${ }^{29}$ The discriminatory potential of RSQMs suggests promise for an assessment method that does not rely on raters. However, RSQMs must be developed and empirically tested in other clinical care environments. The aims of this study were to (1) determine the extent to which previously described consensus methods used in PEM can be used to develop RSQMs for inpatient general internal medicine (GIM) and (2) describe the characteristics of RSQMs developed for inpatient GIM, including how they differ compared with the PEM context.

\section{METHODS}

\section{Selection of Clinical Conditions}

We selected a single rotation, GIM inpatient wards, on which all internal medicine (IM) and medicine-pediatrics (MP) residents rotate. Focusing on a single rotation allowed us to control the scope of the study, and to test the feasibility of generating RSQMs for specific clinical environments. We identified clinical conditions commonly managed on an inpatient GIM rotation for which RSQMs could be developed. Similar to Schumacher et al., we decided that a manageable number (10-15) of RSQMs for each condition would allow for the development of resident performance profiles. ${ }^{24}$ When choosing conditions, we were guided by our previous curricular reform work, during which faculty content experts selected skills that were common and observable on their rotation. ${ }^{30}$ This work resulted in a list of 16 skills for inpatient GIM rotations such as "Initiate antibiotic(s) for pneumonia" and "Manage derangements of potassium." ${ }^{31}$ We reviewed several years of WBA data and chose six commonly assessed conditions. These included diabetes mellitus, hypertension, chronic obstructive pulmonary disease (COPD), hyperkalemia, hypokalemia, and pneumonia. We created a separate category-"Inpatient GIM"- to capture measures that were not specific to the aforementioned conditions, or for measures that were replicated across multiple conditions, indicating they were important across GIM inpatient care rather than specific to one condition.

\section{Generation and Prioritization of Measures}

We followed methods previously described in PEM to generate and prioritize RSQMs. ${ }^{24,25}$ We used the nominal group technique (NGT) to generate candidate measures, followed by a modified Delphi process to prioritize those measures (Fig. 1). ${ }^{32,33}$ Residents and faculty members both completed the NGT and Delphi processes as previous RSQM work showed that each stakeholder group provided unique insights. ${ }^{25}$ We sent recruitment emails to all University of Cincinnati (UC) IM and MP residents and Department of Medicine faculty members, and selected respondents who represented multiple different years in residency training, different faculty clinical roles, and different levels of faculty experience (Table 1). All members had spent multiple weeks on inpatient GIM wards and were considered content experts in the workflow of the rotation.

Nominal Group Technique. We held two NGT meetings, one with nine residents and one with six faculty members (Table 1). The meetings were held separately to avoid power dynamics and fears of sharing "incorrect" responses. Following Schumacher et al.'s methods, ${ }^{24}$ the meeting facilitator (B.K.) asked participants to generate potential quality measures for the six clinical conditions using the following criteria: (1) important to quality care on an inpatient GIM rotation for a patient with the condition of interest and (2) achievement of the measure would likely primarily represent a resident's care for the patient (not another provider). We informed participants that while many quality measures would be extracted from the electronic health record (EHR), they should exclude extraction feasibility from their thought process.

Participants wrote down potential measures without input from others. We then used a round-robin discussion strategy allowing each participant to share ideas with the group. Study team members recorded candidate measures, and participants asked clarifying questions to ensure understanding. We then removed duplicate measures.

Delphi. Similar to the NGT meetings, we used email to recruit UC IM and MP residents and faculty for a single Delphi group. The final group consisted of seven residents and seven faculty members (Table 1 ).

We completed three Delphi rounds via email using an Excel spreadsheet (version 14.7.7, Microsoft, Inc., Redmond, WA) for rating measures each round. Response rate for each round was $100 \%$. In each round, Delphi participants rated measures generated during the preceding NGT meetings on a 6-point scale ( $1=$ lowest, $6=$ highest) for the same two criteria used in the NGT groups: (1) important to quality care on an inpatient GIM rotation and (2) primarily represents a resident's care for the patient. We defined a priori that consensus for inclusion would be met if at least 11/14 participants rated a measure as a 5 or 6 in both criteria. For automatic exclusion, 11/14 participants would need to rate a measure as a 1 or 2 in both criteria. Measures meeting inclusion or exclusion criteria in one round were not rated again in subsequent rounds. In each round, participants made comments that were anonymized and shared with raters in later rounds. Delphi participants did not know the inclusion or exclusion criteria to avoid biased responses.

In rounds 2 and 3, each participant received an individual Excel sheet including measures that had not yet reached inclusion or exclusion criteria, their own previous rating, the 


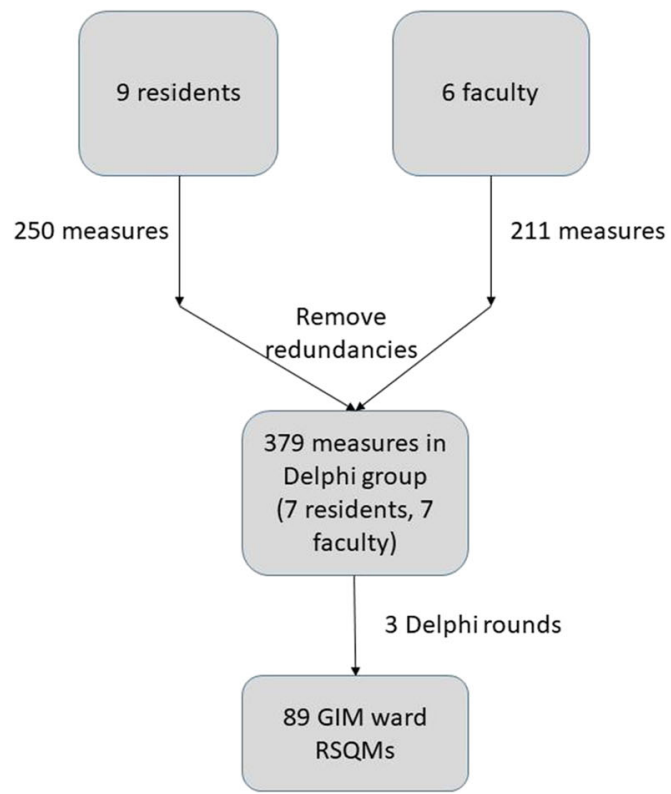

1. Nominal group technique

2. Combining candidate measures

3. Delphi groups

4. Collation of final measures

Figure 1 Overview of the consensus process used to generate inpatient general internal medicine resident-sensitive quality measures.

distribution of the group's previous ratings, and anonymized comments. Participants rated remaining measures considering this collective information as a guide. Consistent with our a priori intent, we ended successive rounds after reaching approximately $10-15$ measures for most conditions.

\section{Final Measure Categorization}

To compare with RSQMs previously developed for PEM, we categorized the final GIM RSQMs as process measures, defined as evidence-based elements of patient care that do not directly assess the patient's clinical condition, ${ }^{34,35}$ or outcome measures. We also detailed the type of work they represented (e.g., documentation vs order vs other), and categorized whether measures would most likely relate to the primary admitting diagnosis, a comorbidity distinct from this diagnosis but requiring management during hospitalization, or both. For

Table 1 Individual Characteristics of Nominal Group Technique (NGT) and Delphi Group Members

\begin{tabular}{llll}
\hline \hline Characteristic & $\begin{array}{l}\text { NGT } \\
\text { resident } \\
\text { group } \\
(\boldsymbol{n}=\mathbf{9})\end{array}$ & $\begin{array}{l}\text { NGT faculty } \\
\text { group }(\boldsymbol{n}=\mathbf{6})\end{array}$ & $\begin{array}{l}\text { Delphi group } \\
(\boldsymbol{n}=7 \text { residents } \\
\text { and 7 faculty } \\
\text { members) }\end{array}$ \\
\hline Gender & $\begin{array}{l}\text { Female-7 } \\
\text { Male-2 }\end{array}$ & $\begin{array}{l}\text { Female-3 } \\
\text { Male-3 }\end{array}$ & $\begin{array}{l}\text { Female-7 } \\
\text { Male-7 }\end{array}$ \\
Specialty & IM-5 & $\begin{array}{l}\text { IM-3 } \\
\text { MP_3 }\end{array}$ & $\begin{array}{l}\text { IM-8 } \\
\text { MP-6 } \\
\text { Ambulatory } \\
\text { practitioners-3 }\end{array}$ \\
role & MP-4 & Ambulatory & $\begin{array}{l}\text { Hospitalists-4 } \\
\text { practitioners-3 }\end{array}$ \\
Experience & N/A & Hospitalists-3 & $\begin{array}{l}\text { Mean years in } \\
\text { practice-9.4 } \\
\text { (range 3-24) }\end{array}$ \\
& PGY1-3 & $\begin{array}{l}\text { Mean years in } \\
\text { practice-6.5 } \\
\text { (range 2-18) }\end{array}$ & $\begin{array}{l}\text { PGY1-3 } \\
\text { PGY2-2 } \\
\text { PGY3-2 }\end{array}$ \\
& PGY3-1 & &
\end{tabular}

$P G Y$, post-graduate year

$P G Y 5=$ chief resident example, "Order COPD bundle for acute COPD exacerbation" is most likely related to a primary admitting diagnosis, while "Order basal/bolus insulin for patients with insulin-dependent diabetes mellitus" could relate to an admitting diagnosis (e.g., diabetic ketoacidosis) or could be a continuation of therapy for diabetes unrelated to the admission reason. The primary author (B.K.) completed initial categorizations, and then, other authors discussed to achieve consensus.

\section{Analysis of Measure Categorization}

We performed a post hoc analysis to investigate why all RSQMs were process measures. We compared mean Delphi group scores between process and outcome measures using the Student $t$ test. We conducted all analyses using SAS (Cary, $\mathrm{NC}$, v. 9.4).

The UC institutional review board deemed this study exempt. NGT and Delphi contributors all signed a document of informed consent and received $\$ 100$ for participating.

\section{RESULTS}

The resident NGT group generated 250 candidate measures, and the faculty NGT group 211. With duplicate measures removed, the final number of candidate measures used for the first Delphi round totaled 379, broken down by condition: diabetes mellitus-73, hypertension-70, COPD-63, hyperkalemia - 24, hypokalemia-21, pneumonia-53, and inpatient GIM-75. Eighty-nine measures reached inclusion consensus (Table 2) at the end of three Delphi rounds, with the following breakdown by condition: inpatient GIM-21, diabetes mellitus - 16, hyperkalemia-14, COPD-13, hypertension - 11, pneumonia-10, and hypokalemia -4 . 
Table 2 The 89 RSQMs for General Internal Medicine Resulting from Consensus-Building Methods

$\overline{\text { Condition }}$ Resident-sensitive quality measure

COPD 1. Document baseline (pre-admission) supplemental oxygen requirements

2. Document changes made to COPD medications during admission in the discharge summary

3. Document daily supplemental oxygen requirement

4. Document daily lung exam for acute COPD exacerbations

5. Document mental status exam for acute COPD exacerbations

6. Document admission respiratory exam for acute COPD exacerbation

7. Document supplemental oxygen requirement present on admission

8. Order albuterol for acute COPD exacerbations

9. Order COPD care bundle for acute COPD exacerbations

10. Order corticosteroids for acute COPD exacerbations

11. Order corticosteroids for 5-day course for acute COPD exacerbation

12. Order no duplicate COPD medications on discharge

Diabetes mellitus

13. Order oxygenation walk test if new inpatient oxygen requirement is persistent at time of discharge

1. Document possible triggers for diabetic ketoacidosis episode

2. Document diabetes classification

3. Document home diabetes medication regimen

4. Order finger stick glucose checks

5. Order finger stick glucose checks every $6 \mathrm{~h}$ for patients with insulin-dependent diabetes mellitus and a diet order that is "nothing by mouth"

6. Order finger stick glucose checks every $6 \mathrm{~h}$ if a patient with insulin-dependent diabetes mellitus is receiving continuous enteral feeds

7. Order finger stick glucose checks with meals if a patient with insulin-dependent diabetes mellitus has an oral diet order

8. Re-order oral diabetes mellitus medications on discharge

9. Adjust insulin dose at least every $24 \mathrm{~h}$ with consistent hyperglycemia

10. Document daily insulin plan in progress note

11. Order "nothing by mouth" initially for patients with diabetic ketoacidosis on an insulin drip

12. Document changes to home insulin regimen in the discharge summary

13. Order basal/bolus insulin for patients with insulin-requiring diabetes mellitus

14. Order correct insulin dose for patients with insulin-requiring diabetes mellitus

15. Order insulin for all patients with type 1 diabetes mellitus

16. Order consult from diabetes educator for all patients with poorly controlled home glucoses or new diagnoses of diabetes mellitus

Hyperkalemia 1. Document a differential diagnosis for etiologies of hyperkalemia

2. Document date of last hemodialysis prior to admission if the patient is dialysis-dependent

3. Document hyperkalemia as a problem in the admission note

4. Document interpretation of any electrocardiogram obtained for hyperkalemia

5. Hold ACE inhibitor medications on admission with hyperkalemia

6. Hold angiotensin receptor blocker medications on admission with hyperkalemia

7. Hold potassium supplements on admission with hyperkalemia

8. Hold potassium-sparing diuretics on admission with hyperkalemia

9. Order a repeat serum potassium lab after any intervention made to lower the potassium.

10. Order an electrocardiogram with severe hyperkalemia

11. Order intravenous calcium if electrocardiogram changes related to hyperkalemia are noted

12. Order potassium binding medication

13. Order repeat serum potassium if the lab sample is noted to be hemolyzed

14. Order telemetry with severe hyperkalemia

Hypokalemia $\quad 1$. If patients receive potassium supplementation during hospitalization, document if continued potassium supplementation is needed at discharge in the discharge summary

2. Order serum magnesium with severe hypokalemia

3. Order serum potassium lab daily if receiving intravenous diuretics

4. Order telemetry with severe hypokalemia

Hypertension 1. Document blood pressure goals with hypertensive urgency/emergency

2. Document cardiovascular exam for hypertensive urgency/emergency

3. Document changes made to antihypertensive medications during admission in the discharge summary

4. Document evaluation for end organ damage when evaluating hypertensive urgency/emergency

5. Document presence or absence of symptoms related to hypertension (e.g., headache, chest pain, dyspnea, visual changes) in patients admitted with hypertensive urgency/emergency

6. Document reasoning in the discharge summary when home antihypertensive medications are discontinued

7. Document substance abuse history for hypertensive urgency/emergency

8. Hold ACE inhibitors with acute kidney injury

9. Hold angiotensin receptor blocker medications with acute kidney injury

10. Hold diuretic medications with non-cardiorenal acute kidney injury

11. Order change to antihypertensive medications if a patient is hypotensive

Pneumonia

1. Document antibiotic(s) used in discharge summary

2. Document baseline (pre-admission) supplemental oxygen requirements

3. Document comorbidities that increase the risk of pneumonia (e.g., immunocompromised state, aspiration)

4. Document daily supplemental oxygen requirement

5. Document risk factors for multi-drug-resistant organisms (e.g., hospitalization of two or more days in the previous

90 days, from nursing home, antibiotic use in the last 90 days)

6. Document supplemental oxygen requirement present on admission

7. Document supplemental oxygen requirement prior to discharge

8. Document total antibiotic duration in discharge summary

9. Order speech therapy evaluation for aspiration pneumonia 
Table 2. (continued)

\begin{tabular}{ll}
\hline \hline Condition & Resident-sensitive quality measure \\
\hline Inpatient general internal & 10. Review chest x-ray images in picture archiving and communication system (PACS) \\
medicine & 1. Complete admission medication reconciliation \\
2. Complete discharge medication reconciliation & 3. Document code status on admission \\
4. Document discharge weight in discharge summary in patients with congestive heart failure \\
5. Document goals of care discussions that result in code status changes \\
6. Document reason for not ordering deep venous thrombosis prophylaxis on admission \\
7. Document source of positive blood culture specimens \\
8. Medications listed in the discharge instructions match the discharge medication reconciliation \\
9. Medications listed in the discharge instructions match the discharge summary \\
10. Medications listed in the discharge summary match the discharge medication reconciliation \\
11. Order alcohol withdrawal order set for acute alcohol withdrawal \\
12. Order code status on admission \\
13. Order daily weights in patients with congestive heart failure exacerbation \\
14. Order deep venous thrombosis prophylaxis on admission \\
15. Order home noninvasive positive pressure ventilation on admission \\
16. Order intravenous proton pump inhibitor for acute gastrointestinal bleed \\
17. Order repeat hemoglobin/hematocrit in patients with active gastrointestinal bleeding \\
18. Order seizure precautions in patients with seizure history or condition placing them at risk of seizure \\
19. Order suicide precautions for patients with self-harm or intentional ingestion \\
20. Order surgical team consult for acute abdomen \\
21. Update sign out document daily
\end{tabular}

All final RSQMs were process measures. Several measures generated by the NGT groups were outcome measures (e.g., "Time with blood pressure in goal range"), but these were not prioritized by the Delphi group. Forty-eight percent (43/89) of the final measures related to documentation (e.g., Document interpretation of EKG), 51\% (45/89) related to orders (e.g., Order finger stick glucose checks), and one measure was categorized as "other" (Review chest x-ray in picture archiving and communication systems). Fifty-eight percent (52/89) of measures were categorized as most likely relating to a primary admitting diagnosis (e.g., Order repeat hemoglobin or hematocrit in patients with acute gastrointestinal bleed), while $42 \%$ (37/89) were categorized as potentially being related to either a primary admitting diagnosis or a comorbidity (e.g., Adjust insulin dose at least every $24 \mathrm{~h}$ with consistent hyperglycemia).

In the post hoc analysis, the average Delphi group scores for process and outcome measures were calculated by adding final round group scores (e.g., maximum score for any measure being $14 \times 6=84$ ) and dividing by the total number of process and outcome measures respectively. Process and outcome measures had similar average group scores for criterion 1 , importance to clinical care (60.7 vs 60.2 respectively, $p=$ 0.85 ), but significantly different scores for criterion 2 , represents a resident's care ( 66.5 vs $49.4, p<0.001)$.

\section{DISCUSSION}

This study used previously described consensus-building methods to generate RSQMs relating to multiple clinical conditions commonly encountered on an inpatient GIM rotation. Competency-based medical education (CBME) has brought increased focus on learner abilities, often measured with WBAs. While this method has the advantage of assessing real-world performance, there are several drawbacks including assessors using variable frames of reference ${ }^{29}$ leading to suboptimal reliability for high-stakes decisions, ${ }^{36-38}$ as well as the challenge of ensuring direct learner observation. ${ }^{39}$ By reducing reliance on raters, RSQMs greatly reduce challenges of rater bias and supplement direct observation to give insight into resident performance.

Once RSQMs are identified and given specific operational definitions, systems to extract and display data could be automated, minimizing resources needed to sustain RSQM use. Compliance targets should be set below $100 \%$ to account for instances when residents deviate from fulfilling these measures for valid reasons (e.g., unique patient situation or clinical context).

\section{Measuring Discrete Tasks}

Developing RSQMs that are discrete tasks rather than broad composite measures holds multiple benefits. Data on discrete tasks may provide specific, formative feedback to learners, while composite measures may lack specificity. Using taskspecific measures also allows for combining measures into different types of composite measures, making possible the assessment of learners through different lenses. For example, condition-specific composite measures could provide information on performance with a given clinical condition, or could be combined to make composite measures for processes across conditions (e.g., admission, discharge, medication ordering).

\section{RSQMs as Process Measures}

All final RSQMs were categorized as process measures, with half representing documentation tasks and half representing orders. Multiple potential reasons exist for why outcome measures produced in the NGT were not prioritized in the Delphi. First, while Delphi group members were instructed to disregard feasibility when prioritizing candidate measures, they 
may have been aware that final RSQMs would most likely be extracted from the EHR. This may have biased members toward more easily obtained process measures such as documentation and orders. Second, Delphi members may have thought that residents are most responsible for process measures, while outcome measures are the collective result of multiple team members (e.g., attendings, nurses), and system and patient factors. This hypothesis is supported by the post hoc analysis showing that outcome measures had lower Delphi group scores for criterion 2 (represents a resident's care) than process measures. However, there is some evidence that residents significantly contribute to outcomes. Denson et al. showed a statistically significant increase in adjusted hospital mortality for IM patients who were discharged within 7 days following a change in resident providers, which occurred independent of attending switches. ${ }^{40}$ A similar study showed increased odds of 30-day and 90-day post-discharge mortality when patients were discharged within 7 days following an intern or resident team transition, which occurred at different times than attending switches. ${ }^{41}$ While these studies do not prove causality, they suggest that the impact of resident care goes beyond process measures and may contribute significantly toward outcomes.

The consequences of using only process measures in resident assessment are unclear. Some studies suggest that better performance on process measures may translate to improved outcomes. ${ }^{35,42-44}$ For example, improved use of a standard COPD care bundle (process measure) has been shown to improve length of stay and readmission rates (outcome measures). ${ }^{45,46}$ However, the association between process and outcomes is not always well-defined ${ }^{34}$ and is dependent on clinical care context, the evidence supporting the process measure, data sources, and other difficult to measure factors. Even so, process measures have value for multiple reasons. First, operationalizing process measures may be more straightforward than outcome measures. ${ }^{35}$ Second, they may be more attributable to individual clinicians given the complex system that contributes to patient outcomes. ${ }^{35}$ Finally, process measures are important for formative organizational and individual efforts, ${ }^{34}$ and providing RSQM data to learners may drive personal and system-wide improvement. Process measures can be a starting point, leading to outcome measures as measurement and attribution techniques improve.

\section{RSQMs and the Relationship to the Primary Admitting Diagnosis}

Forty-two percent of RSQMs in our study related to either to the primary admitting diagnosis or to chronic comorbidities requiring management while patients are admitted for unrelated reasons. This differs from original RSQM work done in PEM, in which nearly all measures directly related to the primary diagnosis. This difference has multiple possible reasons. First, adult patients are more likely than children to have multiple chronic conditions such as hypertension, diabetes, and chronic lung disease, which require active management. Second, GIM inpatients have longer lengths of stay than PEM patients. Our hospital's average length of stay for a GIM inpatient is roughly 4 days, while most PEM visits are a matter of hours. Longer stays mean chronic conditions have a higher chance of requiring active management. Finally, there may be differences in the types of patients who require admission and those seen in the emergency department, such as more comorbidities for those requiring admission. It is unclear if RSQMs are equally valid for assessing residents regardless of whether they are related to the primary admission diagnosis or a chronic comorbidity. It is also unclear if resident contribution to care is the same in these two situations. Perhaps for GIM inpatient teams, attendings focus more on primary diagnoses, leaving management of chronic conditions more to resident purview, and therefore, resident contribution is greater for RSQMs related to chronic conditions. Further studies to develop RSQMs in other specialties and settings are needed to understand what works for whom in which settings, and to understand why.

Our study has several limitations. It was performed at a single residency program, at one institution, in a single clinical setting. The resulting RSQMs are likely influenced by our institution's local workflow, EHR, and practice patterns, and therefore may not generalize to other institutions. Also, while we used our current WBA program to choose conditions for which to build measures, other programs may choose other conditions common to GIM. However, we believe that institutions can follow these methods to develop RSQMs that fit their local systems to measure resident performance for conditions important in local contexts. There is no gold standard to know if the RSQMs produced in this study can provide valid assessment of resident performance. Seeking such evidence comprises important next steps with the RSQMs developed in this study. During consensus building, group members were not specifically told to consider only primary admitting diagnoses, comorbidities, or both, and it is unclear if a shared mental model was truly formed amongst the group in this regard. Further studies are needed to understand if primacy of diagnosis influences resident contribution to outcomes. Finally, residents work in teams and in complex clinical environments. While our goal was to generate measures that are highly attributable to a single learner, there could be team effects that still influence performance on a given RSQM. The attribution/contribution challenge will always exist when measuring performance of an individual in a dynamic, team-based environment. ${ }^{14}$ However, since we graduate, certify, and credential individuals, we must continue to try and find ways to tease out and measure an individual's performance in this complex milieu.

\section{CONCLUSIONS}

We used consensus methods to generate and prioritize 89 RSQMs for a GIM ward rotation at a single institution. All 
of the prioritized RSQMs were process measures, and while it is unclear if higher performance on these measures translates to improved patient outcomes, they likely hold value for purposes of learner assessment, program evaluation, providing formative feedback, and as an initial step toward using outcome measures. Next steps include creating specific operational definitions to determine which RSQMs can be accurately measured using EHR data, testing feasibility of operationalizing RSQMs, building validity evidence for their use in resident assessment, and striving to identify and validate outcome measures for the same purposes.

Corresponding Author: Benjamin Kinnear, MD, MEd; Department of Pediatrics, University of Cincinnati College of Medicine, Cincinnati, OH, USA (e-mail: kinneabn@ucmail.uc.edu).

Funding This work was funded by an Academic Research Committee grant from the Cincinnati Children's Hospital Medical Center Research Foundation.

\section{Compliance with Ethical Standards:}

Conflict of Interest: The authors declare that they do not have a conflict of interest.

\section{REFERENCES}

1. Asch DA, Nicholson S, Srinivas S, Herrin J, Epstein AJ. Evaluating obstetrical residency programs using patient outcomes. Jama. 2009;302(12): 1277-1283.

2. Bansal N, Simmons KD, Epstein AJ, Morris JB, Kelz RR. Using patient outcomes to evaluate general surgery residency program performance. JAMA Surg. 2016;151(2):111-119.

3. Chen C, Petterson S, Phillips R, Bazemore A, Mullan F. Spending patterns in region of residency training and subsequent expenditures for care provided by practicing physicians for Medicare beneficiaries. Jama. 2014;312(22):2385-2393.

4. Sirovich BE, Lipner RS, Johnston M, Holmboe ES. The association between residency training and internists' ability to practice conservatively. JAMA Intern Med. 2014;174(10):1640-1648.

5. Norcini JJ, Boulet JR, Opalek A, Dauphinee WD. Specialty board certification rate as an outcome metric for gme training institutions: a relationship with quality of care. Eval Health Prof. 2020;43(3):143-148.

6. van der Leeuw RM, Lombarts KM, Arah OA, Heineman MJ. A systematic review of the effects of residency training on patient outcomes. BMC Med. 2012;10(1):65.

7. Schumacher DJ, van der Vleuten CP, Carraccio CL. The future of highquality care depends on better assessment of physician performance. JAMA Pediatr. 2016;170(12):1131-1132.

8. Smirnova A, Sebok-Syer SS, Chahine S, et al. Defining and Adopting Clinical Performance Measures in Graduate Medical Education: Where Are We Now and Where Are We Going? Acad Med. 2019;94(5):671-677.

9. Triola MM, Pusic MV. The education data warehouse: a transformative tool for health education research. J Grad Med Educ. 2012;4(1):113-115.

10. Zafar MA, Diers T, Schauer DP, Warm EJ. Connecting resident education to patient outcomes: the evolution of a quality improvement curriculum in an internal medicine residency. Acad Med. 2014;89(10): 1341-1347.

11. Haan CK, Edwards FH, Poole B, Godley M, Genuardi FJ, Zenni EA. A model to begin to use clinical outcomes in medical education. Acad Med. 2008;83(6):574-580.

12. Willett LL, Heudebert GR, Palonen KP, et al. The importance of measuring competency-based outcomes: Standard evaluation measures are not surrogates for clinical performance of internal medicine residents. Teach Learn Med. 2009;21(2):87-93.

13. Gillespie C, Zabar S, Altshuler $\mathbf{L}$, et al. The Research on Medical Education Outcomes (ROMEO) Registry: addressing ethical and practical challenges of using "bigger," longitudinal educational data. Acad Med. 2016;91(5):690-695.

14. Schumacher DJ, Dornoff E, Carraccio C, et al. The Power of Contribution and Attribution in Assessing Educational Outcomes for Individuals, Teams, and Programs. Acad Med. 2020;95(7):1014-1019.

15. Gebauer S, Steele E. Questions program directors need to answer before using resident clinical performance data. J Grad Med Educ. 2016:8(4):507-509.

16. Magraw RM, Fox DM, Weston JL. Health professions education and public policy: a research agenda. J Med Educ. 1978;53(7):539-546.

17. Warm EJ, Mathis BR. Ambulatory Education: Time to Move From Process to Outcome. J Grad Med Educ. 2019;11(2):143-145.

18. Weinstein DF. Optimizing GME by measuring its outcomes. $\mathrm{N}$ Engl J Med. 2017;377(21):2007-2009

19. Triola MM. Hawkins RE, Skochelak SE. The time is now: Using graduates' practice data to drive medical education reform. Acad Med. 2018;93(6):826-828.

20. Chahine $\mathbf{S}$, Kulasegaram KM, Wright $\mathbf{S}$, et al. A call to investigate the relationship between education and health outcomes using big data. Acad Med. 2018;93(6):829-832.

21. Schickedanz A, Gupta R, Arora VM, Braddock III CH. Measuring value in internal medicine residency training hospitals using publicly reported measures. Am J Med Qual. 2018;33(6):604-613.

22. Kalet AL, Gillespie CC, Schwartz MD, et al. New measures to establish the evidence base for medical education: identifying educationally sensitive patient outcomes. Acad Med. 2010;85(5):844-851.

23. Yin HS, Jay M, Maness L, Zabar S, Kalet A. Health literacy: an educationally sensitive patient outcome. J Gen Intern Med. 2015;30(9): 1363-1368.

24. Schumacher DJ, Holmboe ES, van der Vleuten C, Busari JO Carraccio C. Developing resident-sensitive quality measures: A model from pediatric emergency medicine. Acad Med. 2018:93(7):1071-1078.

25. Schumacher DJ, Martini A, Holmboe E, et al. Developing ResidentSensitive Quality Measures: Engaging Stakeholders to Inform Next Steps. Acad Pediatr. 2019;19(2): 177-185

26. Schumacher DJ, Martini A, Holmboe E, et al. Initial Implementation of Resident-Sensitive Quality Measures in the Pediatric Emergency Department: A Wide Range of Performance. Acad Med. 2020;95(8):1248-1255.

27. Schumacher DJ, Holmboe E, Carraccio C, et al. Resident-Sensitive Quality Measures in the Pediatric Emergency Department: Exploring Relationships With Supervisor Entrustment and Patient Acuity and Complexity. Acad Med. 2020;95(8):1256-1264.

28. Schumacher DJ, Martini A. Sobolewski B, et al. Use of ResidentSensitive Quality Measure Data in Entrustment Decision-Making: A Qualitative Study of Clinical Competency Committee Members at One Pediatric Residency. Acad Med. 2020.

29. Kogan JR, Conforti L, Bernabeo E, Iobst W, Holmboe E. Opening the black box of clinical skills assessment via observation: a conceptual model. Med Educ. 2011;45(10): 1048-1060.

30. Warm EJ, Mathis BR, Held JD, et al. Entrustment and mapping of observable practice activities for resident assessment. J Gen Intern Med. 2014:29(8):1177-1182.

31. University of Cincinnati Internal Medicine residency curriculum 20192020. https://www.med.uc.edu/docs/default-source/default-document-library/im-residency-program-curriculum-2019-2020.pdf? sfvrsn=0. Accessed 2020.

32. Humphrey-Murto S, Varpio L, Gonsalves C, Wood TJ. Using consensus group methods such as Delphi and Nominal Group in medical education research. Med Teach. 2017;39(1):14-19.

33. Humphrey-Murto S, Varpio L, Wood TJ, et al. The use of the Delphi and other consensus group methods in medical education research: a review. Acad Med. 2017;92(10):1491-1498.

34. Lazar EJ, Fleischut P, Regan BK. Quality measurement in healthcare Annu Rev Med. 2013;64:485-496.

35. Berenson RA, Pronovost PJ, Krumholz HM. Achieving the potential of health care performance measures. Timely Anal Immed Health Pol. 2013;2013:2.

36. Kelleher M, Kinnear B, Sall $\mathbf{D}$, et al. A Reliability Analysis of Entrustment-Derived Workplace-Based Assessments. Acad Med. 2020;95(4):616-622

37. Roberts C, Shadbolt N, Clark T, Simpson $\mathbf{P}$. The reliability and validity of a portfolio designed as a programmatic assessment of performance in an integrated clinical placement. BMC Med Educ. 2014;14(1):197. 
38. ACGME common program requirements. https://www.acgme.org/ acgmeweb/tabid/429/ProgramandInstitutionalAccreditation/ CommonProgramRequirements.aspx. Accessed 2015.

39. Kogan JR, Hatala R, Hauer KE, Holmboe E. Guidelines: The do's, don'ts and don't knows of direct observation of clinical skills in medical education. Perspect Med Educ. 2017;6(5):286-305.

40. Denson JL, McCarty M, Fang Y, Uppal A, Evans L. Increased mortality rates during resident handoff periods and the effect of ACGME duty hour regulations. Am J Med. 2015;128(9):994-1000.

41. Denson JL, Jensen A, Saag HS, et al. Association between end-ofrotation resident transition in care and mortality among hospitalized patients. Jama. 2016;316(21):2204-2213

42. Werner RM, Bradlow ET. Relationship between Medicare's hospital compare performance measures and mortality rates. Jama. 2006;296(22):2694-2702.

43. Werner RM, Bradlow ET. Public reporting on hospital process improvements is linked to better patient outcomes. Health Aff. 2010;29(7):13191324
44. Jha AK, Orav EJ, Li Z, Epstein AM. The inverse relationship between mortality rates and performance in the Hospital Quality Alliance measures. Health Aff. 2007;26(4):1104-1110.

45. Parikh $\mathbf{R}$, Shah TG, Tandon $\mathbf{R}$. COPD exacerbation care bundle improves standard of care, length of stay, and readmission rates. Int $\mathrm{J}$ Chron Obstruct Pulmon Dis. 2016;11:577.

46. Zafar MA, Panos RJ, Ko J, et al. Reliable adherence to a COPD care bundle mitigates system-level failures and reduces COPD readmissions: a system redesign using improvement science. BMJ Qual Saf. 2017;26(11):908-918.

Publisher's Note: Springer Nature remains neutral with regard to jurisdictional claims in published maps and institutional affiliations. 